\section{Une relation Paneth entre cellules souches et niche intestinale}

Béatrice Romagnolo
Inserm U1016, UMR CNRS 8104, Université Paris-Descartes, 24, rue du Faubourg Saint-Jacques, 75014 Paris, France.

beatrice.romagnolo@inserm.fr
> L'épithélium intestinal fascine les chercheurs par sa capacité incessante à se renouveler tout au long de la vie grâce à l'existence de cellules souches. Audelà de leur implication dans l'homéostasie de ce tissu, ces cellules souches jouent un rôle fondamental dans la régénération et la réparation tissulaires, mais également dans la genèse des cancers intestinaux. Ainsi, il apparaît fondamental de mieux les caractériser ce qui requiert une connaissance précise non seulement des cellules elles-mêmes, mais aussi de leur environnement.

\section{Les cellules souches intestinales de type CBC}

Les cellules souches intestinales ont été difficilement identifiables et font, encore aujourd'hui, l'objet d'un grand nombre de débats. Toutefois, il existe au moins un groupe de cellules souches qui fait consensus dans l'ensemble de la communauté scientifique : les CBC (crypt base columnar cell), dont la contribution à l'homéostasie intestinale a été clairement démontrée [1]. Grâce à de nombreuses avancées au niveau génétique, moléculaire et cellulaire, il a été possible de les caractériser in vivo et ex vivo, de les isoler et d'établir un profil de leur transcriptome à partir des résultats d'analyse de puces à ADN $[1$, 2]. De façon inattendue, ces cellules ont un statut de cellules proliférant activement, tout en possédant toutes les caractéristiques typiques des cellules souches. À chaque division, elles engendrent des progéniteurs qui s'amplifient, puis se différencient dans les différents types cellulaires de l'épithé- lium intestinal. Il existe deux grands lignages cellulaires dans cet épithélium : le type absorptif, représenté par les entérocytes, et le type sécrétoire, auquel correspondent les cellules de Paneth, les cellules en gobelet (goblet cells), entéroendocrines et les cellules Tuft. Ces cellules acquièrent leurs caractéristiques de cellules matures au cours de leur migration le long de l'axe cryptovillositaire, à l'exception des cellules de Paneth qui résident dans le fond des cryptes au contact des cellules souches CBC (Figure 1).

\section{Comment définir la niche intestinale régulant les $\mathrm{CBC}$ ?}

Si le programme intrinsèque des $C B C$ est bien décrit, l'implication des cellules avoisinantes dans le maintien, la régulation et l'autorenouvellement de ces $C B C$ est beaucoup moins bien définie. Ce microenvironnement, que l'on désigne aussi par le terme de niche, associe les facteurs moléculaires et cellulaires qui interagissent avec et régulent les propriétés des CBC. Ainsi, de nombreuses cellules localisées dans le fond des cryptes sont susceptibles de participer à la régulation des $\mathrm{CBC}$, dont les cellules épithéliales adjacentes, les myofibroblastes, les neurones entériques, les cellules endothéliales, les lymphocytes intraépithéliaux de même que d'autres composants comme la membrane basale.

Les premiers modèles considéraient le stroma intestinal comme la principale source de niche [3], mais il a été proposé récemment que les cellules de Paneth, connues pour sécréter des pep- tides antimicrobiens, puissent assurer ce rôle [4]. L'analyse de leur transcriptome à partir de puces à ADN révèle qu'elles expriment différents régulateurs clés impliqués dans la prolifération cellulaire, tels que Wnt3, l'EGF [epidermal growth factor], le TGF- $\alpha$ [transforming growth factor- $\alpha$ ], et DII4 [delta-like protein 4], un ligand des récepteurs Notch. Le contact direct des cellules de Paneth avec les CBC pourrait favoriser le passages de signaux entre ces cellules, dont le signal Wnt qui apparaît fondamental. En effet, la signalisation Wnt/ $\beta$-caténine, par son contrôle de la prolifération et du maintien des cellules souches, est indispensable au renouvellement de l'épithélium intestinal [5, 6]. De plus, des cultures d'organoïdes in vitro ainsi que des modèles génétiques in vivo ont indiqué qu'une perte partielle des cellules de Paneth était associée à une diminution du nombre de $C B C$. Ces observations ont amené les auteurs à proposer que les cellules de Paneth agissent en tant que niche essentielle à la survie et à la prolifération des $C B C$ via leur synthèse de facteurs extrinsèques. Dans ce modèle, les cellules souches génèrent leur propre niche qui leur assure, par un effet paracrine, un rôle protecteur et instructeur. Cette organisation requiert indubitablement la signalisation Wnt/ $\beta$-caténine puisqu'elle est responsable non seulement du maintien et de l'autorenouvellement des $C B C$, mais également de la différenciation des cellules de Paneth $[7,8]$. Cependant, une étude réalisée il y a 15 ans par l'équipe de J.I. Gordon [9] et qui se fondait sur la même stratégie 


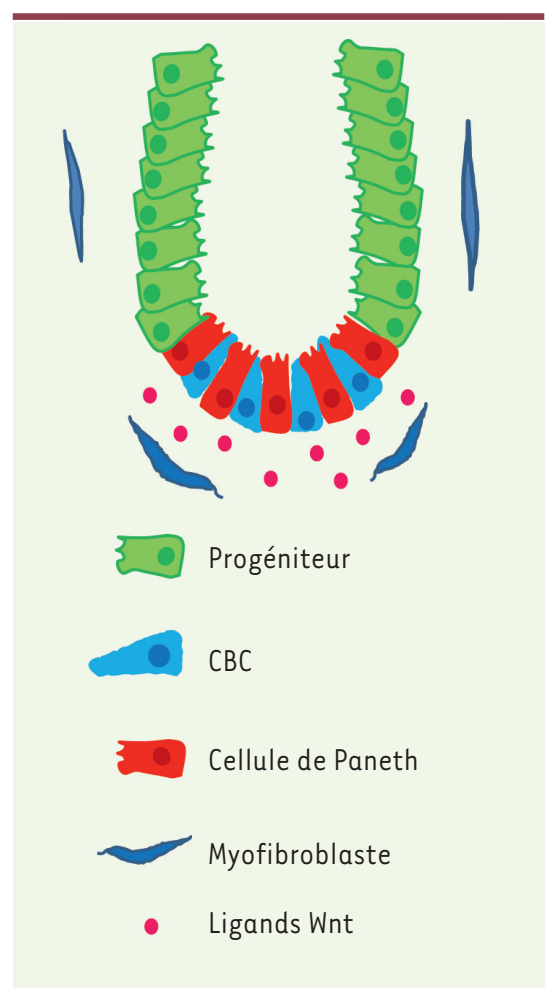

Figure 1. Représentation schématique de la crypte intestinale. Les cellules souches les plus connues et les mieux caractérisées sont les cellules CBC situées au contact immédiat des cellules de Paneth. Ces dernières sécrètent différents facteurs Wnt connus pour stimuler la signalisation $\beta$-caténine nécessaire au maintien et à la prolifération des CBC et des progéniteurs.

d'ablation des cellules de Paneth, avait conduit à des conclusions radicalement opposées, puisqu'aucun effet sur la prolifération de l'épithélium intestinal n'avait été observé.

\section{Quelle contribution pour les cellules} de Paneth à la niche intestinale ?

Compte tenu de cette discordance, il était important de réévaluer la contribution exacte des cellules de Paneth à la niche intestinale. Pour cela, nous avons construit un modèle murin permettant, pour la première fois, l'élimination totale du lignage sécrétoire incluant les cellules de Paneth grâce à la délétion, conditionnelle et inductible par le tamoxifène, du facteur de transcription Mathl [10]. Les souris invalidées pour le gène Mathl sont dépourvues totalement de cellules de Paneth, mais elles ne présentent aucune modification quantitative ou qualitative de leurs cellules souches. Il n'y a pas d'altération de l'expression des marqueurs spécifiques de CBC (0lfm4 [olfactomedin 4], Ascl2 [achaete scute complex homolog 2], Lgr5 [leucine-rich repeat-containing G-protein coupled receptor 5], etc.), et celles-ci conservent leur capacité d'autorenouvellement au cours du temps. Curieusement, l'absence des ligands Wnt, normalement sécrétés par les cellules de Paneth, n'altère pas la signalisation $\beta$-caténine.

Nous avons ensuite évalué les capacités des cellules souches à régénérer l'épithélium intestinal en l'absence de cellules de Paneth dans un contexte de lésion induite. L'épithélium intestinal est très affecté par une forte irradiation. On observe dans les premières heures une apoptose massive suivie d'une prolifération compensatrice permettant la régénération de l'épithélium. Nos travaux indiquent une participation active des cellules souches à la régénération réparatrice, même en l'absence totale de cellules de Paneth.

Enfin, différentes études nous ont conduit à tester l'implication des cellules de Paneth dans un troisième contexte, celui de niche des cellules souches cancéreuses. Des travaux récents de l'équipe de $H$. Clevers ont désigné les CBC comme cellules initiatrices de cancer [11], et nos travaux antérieurs indiquaient l'induction de marqueurs de cellules de Paneth tout au long de la carcinogenèse intestinale $[8,12]$. Nous avons utilisé un modèle murin permettant une invalidation conditionnelle et inductible du gène Apc (adenomatous polyposis coli) dans l'épithélium intestinal, ce qui induit le développement de multiples lésions intestinales [13]. L'analyse de souris invalidées simultanément pour les gènes Apc et Mathl dans l'épithélium intestinal indique que dans ce modèle, les cellules souches sont capables d'initier un processus tumoral malgré l'absence totale de cellules de Paneth avoisinantes. Ainsi, l'ensemble de nos travaux indiquent que les cellules de Paneth ne constituent pas l'unique niche pour assurer le maintien et l'autorenouvellement des cellules souches intestinales, tant dans un contexte physiologique que pathologique.

Afin de démasquer cette autre niche intestinale, nous avons mis en culture des cryptes issues de souris contrôles et de souris déficientes pour le gène Mathl, et comparé leur capacité à proliférer, se renouveler et former des organoïdes. Ces travaux nous ont permis de démontrer que, ex vivo, l'absence des cellules de Paneth - qui ne peut pas être compensée par l'existence d'autres cellules - conduit à une dégénérescence très rapide des cryptes et à une absence totale de formation d'organoïdes. On peut donc conclure que in vivo, en l'absence de Mathl et donc de cellules de Paneth, le signal (ou les signaux) permettant la survie et la prolifération des cellules souches provient(nent) de l'environnement sous-épithélial.

Simultanément à notre publication [10], un article complémentaire à notre étude indique qu'au cours du développement, les CBC sont présentes avant l'apparition des cellules de Paneth et confirment que, chez l'adulte, elles sont capables de proliférer et de se renouveler de façon indépendante des cellules de Paneth [14]. Enfin, il est important de préciser que les cellules de Paneth ne sont présentes que dans l'intestin grêle et pas dans le côlon, confortant donc le concept que d'autres cellules doivent participer à la niche intestinale. L'identité de ces cellules et des signaux qu'elles émettent n'est donc pas encore révélée ; le mystère du couple cellule souche et niche intestinale reste entier... $\diamond$

Paneth cell niche is no longer a driving force

\section{LIENS D'INTÉRÊT}

L'auteur déclare n'avoir aucun lien d'intérêt concernant les données publiées dans cet article. 


\section{RÉFÉRENCES}

1. Barker N, van Es JH, Kuipers J, et al. Identification of stem cells in small intestine and colon by marker gene Lgr5. Nature 2007 ; 449 : 1003-7.

2. Muñoz J, Stange DE, Schepers AG, et al. The Lgr5 intestinal stem cell signature: robust expression of proposed quiescent +4 cell markers. EMBO J 201231 : 3079-91.

3. Powell DW, Pinchuk IV, Saada JI, et al. Mesenchymal cells of the intestinal lamina propria. Annu Rev Physiol $2011 ; 73: 213-37$.

4. Sato T, van Es JH, Snippert HJ, et al. Paneth cells constitute the niche for Lgr5 stem cells in intestinal crypts. Nature $2011 ; 469: 415-8$.

5. Korinek V, Barker N, Moerer P, et al. Depletion of epithelial stem-cell compartments in the small intestine of mice lacking Tcf-4. Nat Genet 1998; 19 : 379-83.
6. Kuhnert F, Davis CR, Wang HT, et al. Essential requirement for Wnt signaling in proliferation of adult small intestine and colon revealed by adenoviral expression of Dickkopf-1. Proc Natl Acad Sci USA $2004 ; 101$ : 266-71.

7. Van Es JH, Jay P, Gregorieff A, et al. Wnt signalling induces maturation of Paneth cells in intestinal crypts. Nat Cell Biol 2005 ; 7 : 381-6.

8. Andreu P, Peignon G, Slomianny C, et al. A genetic study of the role of the Wnt/beta-catenin signalling in Paneth cell differentiation. Dev Biol 2008 ; 324 : 288-96.

9. Garabedian EM, Roberts LJ, McNevin MS, Gordon Jl. Examining the role of Paneth cells in the small intestine by lineage ablation in transgenic mice. J Biol Chem 1997 ; $272: 23729-40$.

10. Durand A, Donahue B, Peignon G, et al. Functional intestinal stem cells after Paneth cell ablation induced by the loss of transcription factor Mathl (Atohl). Proc Natl Acad Sci USA 2012 ; 109 : 8965-70.
11. Barker N, Ridgway RA, van Es JH, et al. Crypt stem cells as the cells-of-origin of intestinal cancer. Nature 2009 ; $457: 608-11$.

12. Palomero T, Lim WK, Odom DT, et al. NOTCHI directly regulates C-MYC and activates a feed-forward-loop transcriptional network promoting leukemic cell growth. Proc Natl Acad Sci USA 2006 ; 103 : 18261-6.

13. Andreu P, Colnot S, Godard C, et al. Crypt-restricted proliferation and commitment to the Paneth cell lineage following Apc loss in the mouse intestine. Development 2005 ; 132 : 1443-51.

14. Kim TH, Escudero $S$, Shivdasani RA. Intact function of Lgr5 receptor-expressing intestinal stem cells in the absence of Paneth cells. Proc Natl Acad Sci USA 2012 ; $109: 3932-7$.

\section{NOUVELLE}

\section{Blocage des fonctions cognitives dans la maladie d'Alzheimer}

\section{Un mécanisme épigénétique dévoilé ?}

Damien Rei
Tsai lab, Picower Institute for learning and memory, Brain and cognitive science department, Massachussetts Institute of Technology (MIT), Cambridge, MA 02139-4 Cambridge, États-Unis.

drei@mit.edu
> La maladie d'Alzheimer (MA) est caractérisée par une déficience progressive des capacités mnésiques et cognitives chez les patients atteints. Les coûts humains et sociétaux associés sont énormes (en 2010 environ 640 milliards de dollars à l'échelle mondiale) et le nombre de personnes atteintes devrait tripler d'ici à 2050. Même si l'effort de recherche est très important, l'étiologie de cette maladie est mal comprise et aucun traitement efficace n'existe encore. Une des hypothèses privilégiées est celle de la voie amyloïde où le clivage de la protéine précurseur de l'amyloïde (APP) provoque la génération du peptide $\beta$-amyloïde $(A \beta)$, responsable de la mort neuronale associée à la maladie, et ce notamment dans les zones du cerveau impliquées dans la mémoire. Une des structures particulièrement affectées par la maladie est l'hippocampe qui est située dans le lobe temporal médian et est impliquée dans la formation de la mémoire épisodique (autobiographique).

\section{Épigénétique et maladie d'Alzheimer : premières suspicions}

Nous avons récemment montré, dans une étude publiée dans Nature en mars 2012 [1], qu'un des mécanismes également en cause dans cette maladie est d'origine épigénétique, et que les modifications ainsi engendrées semblent réversibles. Une modification épigénétique de l'ADN agit sur l'expression des transcrits et donc des protéines sans changer la séquence d'ADN codante. Autrement dit, il n'y a pas de mutation génétique et seule l'accessibilité du gène est modifiée, avec comme corollaire une augmentation ou une diminution de son expression. Plusieurs familles d'enzymes sont responsables de ces modifications et notamment les histones déacétylases (HDAC). Ces dernières comprennent plusieurs membres et régulent de façon négative le processus d'acétylation de la chromatine (d'où leur nom). À l'inverse, les histones acétyltransférases (HAT) sont responsables de l'acétylation de la chromatine. Les groupements acétyl étant chargés négativement, l'acétylation par les HAT induit une structure plus ouverte de la chromatine ce qui, par conséquent, accroît l'accessibilité des gènes à la machinerie de transcription. Au contraire, les HDAC éliminant ces groupements, condensent la chromatine et diminuent l'expression des gènes concernés (Figure 1).

Cette découverte de l'intervention d'un mécanisme épigénétique dans la maladie d'Alzheimer fait suite à une série d'avancées au cours de ces dernières années dans la compréhension du rôle 